\title{
TITLE:
}

\section{Hydration state inside HeLa cell monolayer investigated with terahertz spectroscopy}

\author{
$\operatorname{AUTHOR}(\mathrm{S}):$ \\ Shiraga, K.; Suzuki, T.; Kondo, N.; Tanaka, K.; \\ Ogawa, Y.
}

\section{CITATION:}

Shiraga, K....[et al]. Hydration state inside HeLa cell monolayer investigated with terahertz spectroscopy. Applied Physics Letters 2015, 106(25): 253701.

\section{ISSUE DATE:}

2015-06-23

URL:

http://hdl.handle.net/2433/201614

\section{RIGHT:}

(c) 2015 American Institute of Physics. This article may be downloaded for personal use only. Any other use requires prior permission of the author and the American Institute of Physics. 


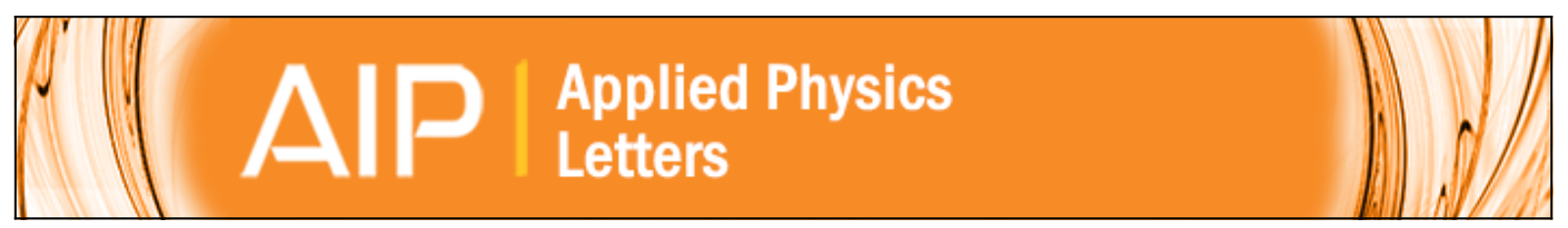

\section{Hydration state inside HeLa cell monolayer investigated with terahertz spectroscopy}

K. Shiraga, T. Suzuki, N. Kondo, K. Tanaka, and Y. Ogawa

Citation: Applied Physics Letters 106, 253701 (2015); doi: 10.1063/1.4922918

View online: http://dx.doi.org/10.1063/1.4922918

View Table of Contents: http://scitation.aip.org/content/aip/journal/apl/106/25?ver=pdfcov

Published by the AIP Publishing

\section{Articles you may be interested in}

Hydration and hydrogen bond network of water around hydrophobic surface investigated by terahertz spectroscopy

J. Chem. Phys. 141, 235103 (2014); 10.1063/1.4903544

Observation of salt effects on hydration water of lysozyme in aqueous solution using terahertz time-domain spectroscopy

Appl. Phys. Lett. 103, 173704 (2013); 10.1063/1.4826699

Determination of the complex dielectric constant of an epithelial cell monolayer in the terahertz region Appl. Phys. Lett. 102, 053702 (2013); 10.1063/1.4790392

Real-time sonoporation through HeLa cells

AIP Conf. Proc. 1474, 271 (2012); 10.1063/1.4749348

Label-free monitoring of interaction between DNA and oxaliplatin in aqueous solution by terahertz spectroscopy Appl. Phys. Lett. 101, 033704 (2012); 10.1063/1.4737401

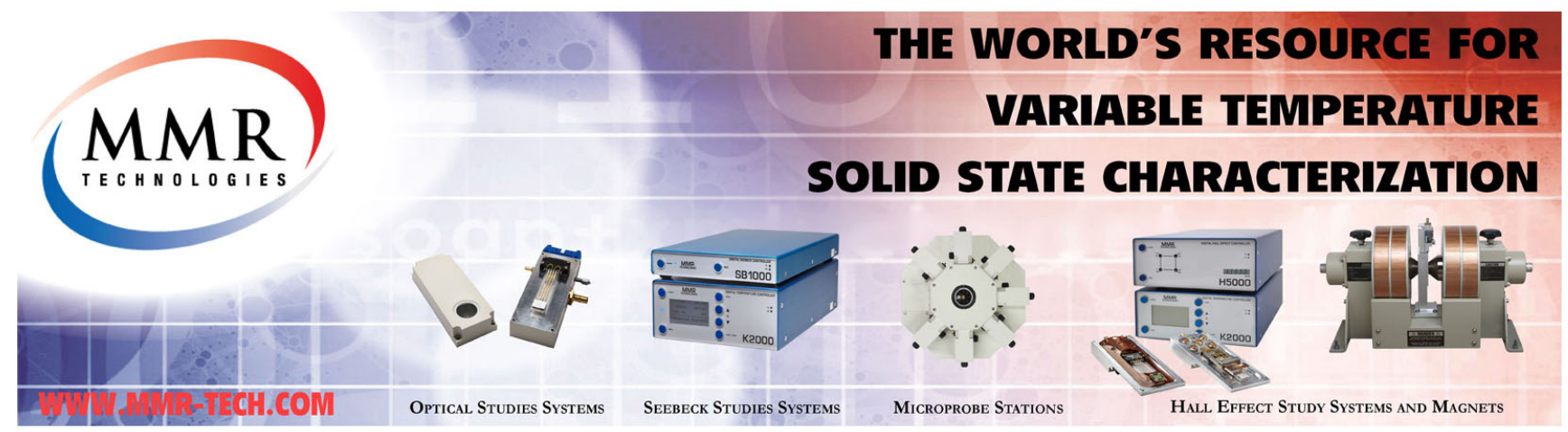




\title{
Hydration state inside HeLa cell monolayer investigated with terahertz spectroscopy
}

\author{
K. Shiraga, ${ }^{1}$ T. Suzuki, ${ }^{1}$ N. Kondo, ${ }^{1}$ K. Tanaka,${ }^{2}$ and Y. Ogawa ${ }^{1, a)}$ \\ ${ }^{1}$ Graduate School of Agriculture, Kyoto University, Kyoto 606-8502, Japan \\ ${ }^{2}$ Department of Physics, Kyoto University, Kyoto 606-8502, Japan
}

(Received 13 March 2015; accepted 11 June 2015; published online 23 June 2015)

\begin{abstract}
The hydration state in living cells is believed to be associated with various cellular activities. Nevertheless, in vivo characterization of intracellular hydration state under physiological condition has not been well documented to date. In this study, the hydration state of an intact HeLa cell monolayer was investigated by terahertz time-domain attenuated total reflection spectroscopy. Combined with the extended theory of Onsager, we found $23.8 \pm 7.4 \%$ of HeLa intracellular water was hydrated to biomolecules (corresponding to $1.25 \mathrm{~g} \mathrm{H}_{2} \mathrm{O} / \mathrm{g}$ solute); exhibiting slower relaxation dynamics than bulk water. (C) 2015 AIP Publishing LLC. [http://dx.doi.org/10.1063/1.4922918]
\end{abstract}

Liquid water, sometimes referred to as the "matrix of life," orchestrates the expression of biological macromolecules, which determines the functional and metabolic state of living cells. In particular, small changes in protein conformation can be associated with cascading effects on the surrounding water molecules: if the conformational change ties up more hydration water (dynamically retarded water in the vicinity of biomolecules), the protein structure becomes more rigid and, at the same time, the diffusion in the surrounding medium is retarded, conversely, if more bulk water is released, the conformation becomes more mobile and the fluidity of the surrounding medium increases. ${ }^{1}$ Simultaneously, the hydration state of the intracellular biomolecules themselves influences the surrounding chemical interactions as well as their own conformational stability, because the net rate of metabolic processes within the cell depends on the diffusion rate of molecules. ${ }^{2}$ In turn, we can assume that the intracellular hydration state will influence, to a degree, the activity of the cell itself. In fact, it has been proposed that "osmotically unresponsive and viscous" hydration water predominates in a resting cell, however, when "reactive and fluid" bulk water becomes more abundant, the cell will switch to an active one. ${ }^{3,4}$ This hypothesis seems to be consistent with previous nuclear magnetic resonance (NMR) results that suggest water in cancerous ( $=$ active) tissues is characterized by increased fluidity compared to that in normal (= stable) tissues, ${ }^{5}$ indicating that cellular activity and pathological states are closely associated with intracellular hydration states.

Spectroscopic methods, such as NMR, ${ }^{6}$ quasi-elastic neutron scattering (QENS), ${ }^{7,8}$ and incoherent neutron scattering (INS) ${ }^{9-11}$ have been used in the last decade to quantitatively determine the hydration state of monocellular organisms (i.e., Escherichia coli and Haloarcula marismortui) and human red blood cells. These studies show that $85 \%-90 \%$ of intracellular water behaves in a bulk-like fashion, while the remaining water molecules (classified as hydration water) exhibit more retarded reorientation and translation dynamics than that of bulk water. However, since

\footnotetext{
${ }^{\text {a) }}$ Author to whom correspondence should be addressed. Electronic mail: ogawayu@kais.kyoto-u.ac.jp
}

the organelles and chemical composition of these cells are substantially different from those of general eukaryotic cells, the present understanding of intracellular hydration cannot be said to have reached a consensus. Furthermore, current in vivo estimation of hydration state is impeded by two issues: first, these techniques cannot spatially distinguish between intracellular and extracellular water. Consequently, any extracellular water within the sampling window will be included in the measurement and obscure, to a degree, the measurement of the "true" intracellular hydration state. Second, hydrogen/deuterium (H/D) substitution or cryogenic cooling is needed in these methods, thus, the "native" hydration state in the cell interior is not directly measured. To overcome these difficulties, nonlinear optical Kerr effect (OKE) microspectroscopy, which probes only a spatially confined volume $(0.5 \mu \mathrm{m}$ in the lateral direction and $4.0 \mu \mathrm{m}$ in the axial coordination) inside a single living cell, has been employed without the need for H/D substitution or cryogenic cooling of the target mammalian culture cell. ${ }^{12}$ As a result, it was shown that the water inside an intact mammalian culture cell under physiological conditions exhibited a 1.7 times slower structural relaxation than that of pure water. This data indicates the mobility of intracellular water is, on average, moderately restricted due to the presence of biological macromolecules and hydration water. However, as far as intracellular water is concerned, firm conclusions cannot yet be drawn due to insufficient experimental evidence. ${ }^{13}$

Recently, terahertz (THz) spectroscopy has attracted attention as a method for determining hydration state. ${ }^{14-17}$ Dielectric responses to the $\mathrm{THz}$ waves, whose periodic electric field corresponds to the lifetime of bulk water hydrogen bonds, ${ }^{18}$ are dominated by relaxational dynamics of bulk water molecules. On the other hand, the relaxation motion of hydration water, which lies below the $\mathrm{GHz}$ frequencies, has negligible contribution in the $\mathrm{THz}$ region. ${ }^{14}$ Accordingly, the dielectric responses in the $\mathrm{THz}$ region are selectively sensitive to bulk water, consequently, any decreases in the $\mathrm{THz}$ signal one can be use to evaluate the hydration state of cells without the need for any preprocessing such as H/D substitution. Additionally, the long wavelength and low photon energy of $\mathrm{THz}$ waves $(300 \mu \mathrm{m}$ and $4 \mathrm{meV}$ at $1 \mathrm{THz}$, 
respectively), relative to those in the optical frequency region, allow one to observe whole living cells (typical cell size: several tens $\mu \mathrm{m}$ in the planar diameter and several $\mu \mathrm{m}$ in the thickness) under physiological conditions. ${ }^{19,20}$ In this study, therefore, we characterized the spatially averaged hydration state inside living culture cells (HeLa, human cervical carcinoma); a measurement based on the complex dielectric constant in the $\mathrm{THz}$ region of the in vivo HeLa cell monolayer.

In order to determine the complex dielectric constant of the cultured HeLa cells, THz time-domain attenuated total reflection spectroscopy (THz TD-ATR) was employed. ${ }^{21}$ The ATR geometry allows one to accurately measure the complex dielectric constants of samples with strong absorption in the THz region, such as water-rich living cells, compared to transmission or external reflection geometries. ${ }^{22}$ The HeLa cells were cultured, in a minimum essential medium (MEM), on a silicon ATR prism of a THz TD-ATR spectrometer, TAS7500 (ADVANTEST Co., Ltd.). The cell incubation chamber was set up on top of the ATR prism, and maintained at $310 \mathrm{~K}$ and supplied with a humidified $5 \% \mathrm{CO}_{2}$ atmosphere. The growth of the cultured cells was observed through the transparent lid of the incubation chamber with an optical microscope. After confirming that the HeLa culture cells had formed a monolayer on the ATR prism, the THz TD-ATR measurements were performed over the range of 0.25 and $3.5 \mathrm{THz}$. More than $4.0 \times 10^{4}$ scans were carried out within $75 \mathrm{~min}$, and to confirm the repeatability, four different samples were measured on different days and then averaged. In our measurements, the standard errors in the real and imaginary part of the complex dielectric constant were at most 0.020 and 0.025 below $1.0 \mathrm{THz}$, and 0.090 and 0.045 between 1.0 and $3.5 \mathrm{THz}$, respectively.

The observed complex dielectric constant of HeLa cells in the MEM is compared with that of distilled water and the MEM in Figure 1. We found the dielectric responses of the MEM were unchanged from those of distilled water, but HeLa cells in the MEM can be distinguished from the MEM. In our THz TD-ATR setup, the localization depth of the evanescent field (estimated to be $23 \mu \mathrm{m}$ at $1.0 \mathrm{THz}$ when the sample is water ${ }^{23}$ ) is greater than the thickness of the HeLa cell monolayer $\left(7.5 \pm 1.0 \mu \mathrm{m}^{20}\right)$. Therefore, the complex dielectric constant of HeLa cells in the MEM in Figure 1 reflected both the dielectric responses of HeLa cells (directly adhered to the ATR prism) and the MEM (on the top of HeLa cells). To determine the complex dielectric constant of the HeLa cell monolayer alone, removing the contribution of the MEM above the HeLa cells, a two-interface model consisting of the ATR prism-HeLa monolayer and HeLamedium boundaries (see the schematic illustration in the inset of Figure 1). In this calculation, the non-uniformity $( \pm 1.0 \mu \mathrm{m})$ in the HeLa cell monolayer interface was taken into account by assuming that the cell monolayer is flat $7.5 \pm 1.0 \mu \mathrm{m}$. Since the (complex) dielectric constants of the ATR prism and the MEM as well as the incident angle and the thickness of the cell monolayer are known, the complex dielectric constant of the HeLa cell monolayer can be calculated theoretically. Details of the calculation process are presented in earlier papers. ${ }^{19,20}$

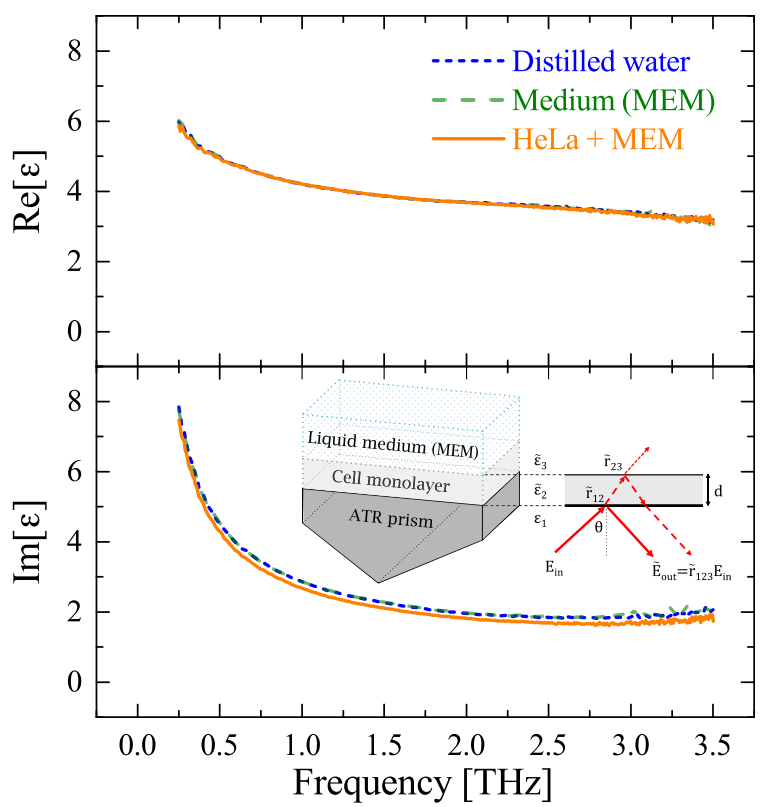

FIG. 1. Complex dielectric constant of distilled water, MEM, and HeLa cells in MEM. The inset shows a schematic illustration of the two-interface model: $\varepsilon_{1}, \tilde{\varepsilon}_{2}, \tilde{\varepsilon}_{3}$ (dielectric constant of the ATR prism, HeLa cell monolayer, and the MEM), $\tilde{r}_{12}, \tilde{r}_{23}$ (Fresnel's reflection coefficient at each boundary), $E_{\text {in }}, \tilde{E}_{\text {out }}$ (incident and reflected $\mathrm{THz}$ electric field), $\theta$ (incident angle), and $d$ (thickness of HeLa cell monolayer).

Figure 2 shows the complex dielectric constants of distilled water, $\tilde{\varepsilon}_{\text {water }}(\omega)$, and the HeLa cell monolayer, $\tilde{\varepsilon}_{H e L a}(\omega)$, at $310 \mathrm{~K}$, between 0.25 and $3.5 \mathrm{THz} . \tilde{\varepsilon}_{H e L a}(\omega)$ accords well with the reported extrapolated data of the complex dialectic constant of human tissues measured at $310 \mathrm{~K} \cdot{ }^{24}$ The insets of Figure 2 represent $\delta \tilde{\varepsilon}(\omega)=\tilde{\varepsilon}_{\text {water }}(\omega)$ $-\tilde{\varepsilon}_{H e L a}(\omega)$, where the error bars in the insets are due to heterogeneity in cellular thickness $(7.5 \pm 1.0 \mu \mathrm{m})$ in the twointerface calculation procedure. As previous studies have suggested, biomolecules dispersed in aqueous solution have

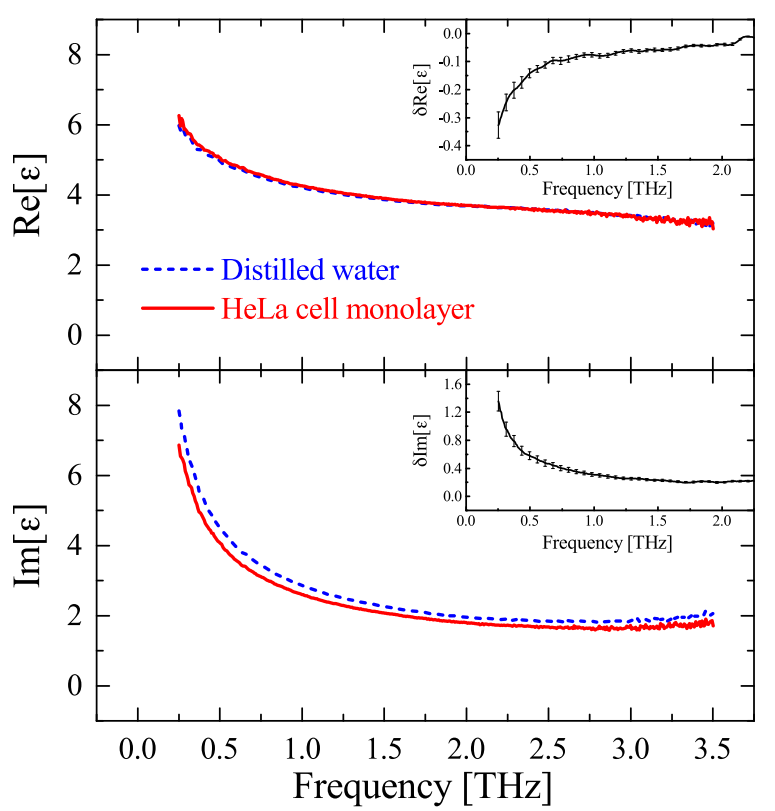

FIG. 2. Comparison of the complex dielectric constants: HeLa cell monolayer $\tilde{\varepsilon}_{H e L a}(\omega)$ and distilled water $\tilde{\varepsilon}_{\text {water }}(\omega)$. The insets are the difference in the complex dielectric constants $\delta \tilde{\varepsilon}(\omega)=\tilde{\varepsilon}_{\text {water }}(\omega)-\tilde{\varepsilon}_{H e L a}(\omega)$. 
negligible absorption in the $\mathrm{THz}$ region. ${ }^{14-16,25}$ This means that the complex dielectric constants of distilled water and HeLa cell monolayer between 0.25 and $3.5 \mathrm{THz}$ come from Debye-type relaxation and Lorentz-type vibration dynamics of the water molecules and can be expressed as a superposition of the complex susceptibilities of slow relaxation $\tilde{\chi}_{\text {slow }}(\omega)$ (collective reorientation of hydrogen-bonded bulk water, $\sim 0.02 \mathrm{THz}$ ), fast relaxation $\tilde{\chi}_{\text {fast }}(\omega)$ (individual reorientation of non-hydrogen-bonded water, $\sim 0.5 \mathrm{THz}$ ), and intermolecular stretching vibration $\tilde{\chi}_{S}(\omega)$ (hindered translation of water, $\sim 5 \mathrm{THz}) .{ }^{26}$ Among these, the slow relaxation mode of bulk water $\left(\tau_{\text {slow }} \approx 6.33\right.$ ps at $\left.310 \mathrm{~K}\right)$ is distinguishable from that of hydration water. This is because the relaxation time of hydration water molecules is much longer than that of bulk water, thereby the contribution of the hydration water relaxation is negligible above $0.25 \mathrm{THz} .{ }^{14}$ In contrast, $\tilde{\chi}_{\text {fast }}(\omega)$ and $\tilde{\chi}_{S}(\omega)$ at sub-picosecond timescales are still present among hydration water molecules since such short-lived water dynamics are less influenced by the hydration effect.

Even though several dielectric functions have been recently demonstrated to describe the terahertz dielectric functions of living objects, ${ }^{20,27}$ it was quite difficult to determine the intracellular hydration state on the basis of these model functions, in principle. Arikawa et al. developed a model based on the theory of Onsager, which takes into consideration the local electric field, to determine the hydration parameters for mono-solute aqueous solutions, where water molecules that no longer exhibit the slow relaxation $\tilde{\chi}_{\text {slow }}(\omega)$ are defined as hydration water. ${ }^{14}$ In this study, aiming to characterize the intracellular hydration state, we extended Arikawa's model to a multi-solute system, where four kinds of biomolecules (protein, lipid, RNA, and DNA) are assumed to be responsible for the hydration state inside the HeLa cell monolayer. In this analysis, $\widetilde{\varepsilon}_{H e L a}(\omega)$-determined by experiment — was decomposed into the complex susceptibilities of bulk water $\tilde{\chi}_{\text {bulk }}(\omega)$, hydration water $\tilde{\chi}_{\text {hyd }}(\omega)$, and biomolecular solutes $\tilde{\chi}_{\text {sol }}(\omega)$, via Eq. (1) using a least-square method

$$
\begin{aligned}
& \tilde{\varepsilon}_{\text {HeLa }}(\omega)-1=\tilde{\chi}_{\text {bulk }}(\omega)+\tilde{\chi}_{\text {hyd }}(\omega)+\tilde{\chi}_{\text {sol }}(\omega) \\
&= \frac{4 \pi\left(N_{\mathrm{w}}-n_{\mathrm{h}} \sum N_{\mathrm{s}}\right) \tilde{g}(\omega)}{1-\tilde{\alpha}_{\text {vib. }}(\omega) \tilde{h}(\omega) / r_{\mathrm{w}}{ }^{3}} \\
& \times\left\{\tilde{\alpha}_{\mathrm{vib} .}(\omega)+\frac{\tilde{\alpha}_{\text {slow }}(\omega)+\tilde{\alpha}_{\text {fast }}(\omega)}{1-\alpha_{\mathrm{w}} h / r_{\mathrm{w}}{ }^{3}}\right\} \\
&+\frac{4 \pi n_{\mathrm{h}} \sum N_{\mathrm{s}} \tilde{g}(\omega)}{1-\tilde{\alpha}_{\mathrm{vib} .}(\omega) \tilde{h}(\omega) / r_{\mathrm{h}}{ }^{3}} \\
& \times\left\{\tilde{\alpha}_{\mathrm{vib} .}(\omega)+\frac{\tilde{\alpha}_{\text {fast }}(\omega)}{1-\alpha_{\mathrm{w}} h / r_{\mathrm{h}}{ }^{3}}\right\} \\
&+\sum_{\mathrm{s}=\mathrm{p}, 1, \mathrm{R}, \mathrm{D}} \frac{4 \pi N_{\mathrm{s}} \tilde{g}(\omega)}{1-\alpha_{\mathrm{s}} \tilde{h}(\omega) / r_{\mathrm{s}}{ }^{3}} \alpha_{\mathrm{s}}
\end{aligned}
$$

where, $n_{\mathrm{h}}$ is the number of hydration water molecules per solute molecule, $r_{\mathrm{w}(\mathrm{h}, \mathrm{s})}$ is the molecular radius of bulk water (hydration water, solute), and $N_{\mathrm{w}(\mathrm{s})}$ is the number density of water (solute). $\sum N_{\mathrm{s}}$ is the sum of the number density of proteins $\left(N_{\mathrm{p}}\right)$, lipids $\left(N_{\mathrm{l}}\right)$, RNA $\left(N_{\mathrm{R}}\right)$, and DNA $\left(N_{\mathrm{D}}\right)$, $\sum N_{\mathrm{s}}=N_{\mathrm{p}}+N_{\mathrm{l}}+N_{\mathrm{R}}+N_{\mathrm{D}}$ (hereafter, the subscripts of "p," "l," "R," and "D" represent proteins, lipids, RNA, and DNA, respectively). Here, the frequency-dependent polarizability volumes of slow relaxation $\tilde{\alpha}_{\text {slow }}(\omega)$, fast relaxation $\tilde{\alpha}_{\text {fast }}(\omega)$, and vibration $\tilde{\alpha}_{\text {vib. }}(\omega)$ were calculated from $\tilde{\chi}_{\text {slow }}(\omega), \tilde{\chi}_{\text {fast }}(\omega)$, and $\tilde{\chi}_{S}(\omega)$ that were noted in our previous paper. ${ }^{20}$ Polarizability volume of the solute $\left(\alpha_{\mathrm{s}}\right)$ was assumed to be a frequency-independent real number because there is no frequency dispersion of solutes in aqueous solution. ${ }^{14} \tilde{g}(\omega)=3 \tilde{\varepsilon}_{H e L a}(\omega) /\left\{2 \tilde{\varepsilon}_{H e L a}(\omega)+1\right\}$ and $\tilde{h}(\omega)=2\left\{\tilde{\varepsilon}_{H e L a}(\omega)-1\right\} /\left\{2 \tilde{\varepsilon}_{H e L a}(\omega)+1\right\}$ are the local correlation terms for the external electric field and reaction electric field, respectively. $\alpha_{w}$ is the high frequency limit $(\omega \rightarrow \infty)$ of $\tilde{\alpha}_{\text {vib. }}(\omega)$, while $h$ is the zero frequency limit $(\omega \rightarrow 0)$ of $\tilde{h}(\omega)$. Furthermore, the volume conservation law

$$
\frac{4 \pi r_{\mathrm{w}}{ }^{3}}{3}\left(N_{\mathrm{w}}-n_{\mathrm{h}} \sum N_{\mathrm{s}}\right)+\frac{4 \pi r_{\mathrm{h}}{ }^{3}}{3} n_{\mathrm{h}} \sum N_{\mathrm{s}}+\sum \frac{4 \pi r_{\mathrm{s}}^{3}}{3} N_{\mathrm{s}}=1
$$

is simultaneously solved with Eq. (1). For a successful fit, the molecular radius of the solutes $\left(r_{\mathrm{s}}\right)$ was fixed (assumed to be spherical in shape), which can be calculated by

$$
r_{\mathrm{s}}=\sqrt[3]{\frac{3}{4 \pi} \frac{M}{N_{\mathrm{A}} \rho}}
$$

where $M$ is the molecular weight, $N_{\mathrm{A}}$ is the Avogadro number, and $\rho$ is the density. At the same time, the polarizability volume of the solutes $\left(\alpha_{s}\right)$ was theoretically calculated from the high-frequency dielectric constant of pure solutes via the Clausius-Mossotti equation, ${ }^{28}$ and treated as fixed values. Furthermore, to increase the number of fixed parameters, the number densities of solute molecules were expressed as a function of $N_{\mathrm{w}}$, based on the fraction of number density $\left(N_{\mathrm{w}}: N_{\mathrm{p}}: N_{\mathrm{l}}: N_{\mathrm{R}}: N_{\mathrm{D}}\right)$, which was estimated by the mass percent and average molar weight of each component inside HeLa cells. All the cited fixed parameters are listed in Table I. These fixed parameters were substituted into Eqs. (1) and (2) to determine the remaining three fitting parameters $\left(n_{\mathrm{h}}, N_{\mathrm{w}}\right.$, and $r_{\mathrm{h}}$ ) via a least-square fitting process. Since living cells may have non-negligible individual differences in cellular volume and chemical components, the uncertainty of the best-fitted parameters has to be considered carefully. In this study, $\tilde{\varepsilon}_{H e L a}(\omega)$ with errors, originating from both experiment and uncertainty in cellular thickness $(7.5 \pm 1.0 \mu \mathrm{m})$, was substituted in the left-hand side of Eq. (1). Additionally, all molecular radii $\left(r_{\mathrm{p}}, r_{\mathrm{l}}, r_{\mathrm{R}}\right.$, and $\left.r_{\mathrm{D}}\right)$ and polarizability volumes $\left(\alpha_{p}, \alpha_{1}, \alpha_{R}\right.$, and $\left.\alpha_{D}\right)$ were varied by $\pm 10 \%$ in order to consider the uncertainty of the fixed parameters in the righthand side of Eq. (1).

The resulting decomposition of $\tilde{\varepsilon}_{H e L a}(\omega)$ into $\tilde{\chi}_{\text {bulk }}(\omega)$, $\tilde{\chi}_{\text {hyd }}(\omega)$, and $\tilde{\chi}_{\text {sol }}(\omega)$ is shown in Figure 3 , together with the fitted parameters $\left(n_{\mathrm{h}}, N_{\mathrm{w}}\right.$, and $\left.r_{\mathrm{h}}\right)$. Figure 3 clearly indicates that the complex dielectric constant of the HeLa cell monolayer was fitted with the fitted parameters $n_{\mathrm{h}}=293.6 \pm 19.6$, $N_{\mathrm{w}}=0.0312 \pm 0.002 / \AA^{3}$, and $r_{\mathrm{h}}=1.78 \pm 0.03 \AA$. Note that $\pm 10 \%$ variance in the fixed molecular radii and polarizability volumes did not significantly affect the decomposed 
TABLE I. Fixed parameters used in the fitting procedure.

\begin{tabular}{lccccc}
\hline \hline & Water & Protein & Lipid & RNA $^{\text {a }}$ & DNA \\
\hline Mass percent $(\%)^{\mathrm{b}}$ & 84 (Ref. 29) & 11 (Ref. 29) & 2.5 (Ref. 30) & 0.4 (Refs. 31 and 32) & $0.2($ Refs. 31 and 33) \\
Molecular weight $M(\mathrm{~g} / \mathrm{mol})$ & 18 & $5.0 \times 10^{4}$ (Ref. 34) & $7.0 \times 10^{2}$ (Ref. 35) & $2.5 \times 10^{6}$ (Ref. 36) & $2.2 \times 10^{12}($ Ref. 37) \\
Normalized mole fraction & 1 & $4.71 \times 10^{-5}$ & $7.65 \times 10^{-4}$ & $3.43 \times 10^{-8}$ & $1.95 \times 10^{-14}$ \\
Density $\rho \times 10^{-24}\left(\mathrm{~g} / \AA^{3}\right)$ & 0.99 & 1.37 (Ref. 38) & 1.01 (Ref. 39) & 1.56 (Ref. 40) & $1.70($ Ref. 41$)$ \\
Molecular radius $r_{\mathrm{s}}(\AA)$ & 1.9 & 24.4 & 6.4 & 79.8 & $9.2 \times 10^{3}$ \\
Polarizability $\alpha\left(\AA^{3}\right)$ & 3.8 (Ref. 14) & $5.6 \times 10^{3}$ (Ref. 42) & 96 (Ref. 15) & $1.2 \times 10^{5}$ (Ref. 42) & $1.9 \times 10^{11}($ Ref. 42) \\
\hline \hline
\end{tabular}

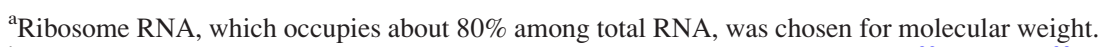

${ }^{\mathrm{b}}$ For RNA and DNA, mass percent was determined by dividing intracellular RNA ${ }^{32}$ and DNA ${ }^{33}$ mass by HeLa cell mass. ${ }^{31}$

frequency dispersions of $\tilde{\chi}_{\text {bulk }}(\omega), \tilde{\chi}_{\text {hyd }}(\omega)$, and $\tilde{\chi}_{\text {sol }}(\omega)$. Although absorption coefficients of macromolecular powders (i.e., $100 \%$ protein powder samples) are around $10-50 \mathrm{~cm}^{-1}$ in the $\mathrm{THz}$ region, ${ }^{42,43}$ our rough estimation showed that their absorption coefficient of proteins weighted by their molar-number in the HeLa cells (i.e., molar-number of intracellular protein is estimated to be about 9 times smaller than that of $100 \%$ protein powder) was approximately 50-200 times smaller than that of water. Since biomolecular $\mathrm{THz}$ absorptions in HeLa cells are expected to have the same order of measurement error, this validates a $\operatorname{Im}\left[\tilde{\chi}_{\text {sol }}(\omega)\right]$ of approximately 0 , as shown in Figure 3 .

Based on the number densities $\left(N_{\mathrm{p}}=4.7 \times 10^{-5} N_{\mathrm{w}}\right.$, $N_{\mathrm{l}}=7.7 \times 10^{-4} N_{\mathrm{w}}, N_{\mathrm{R}}=3.4 \times 10^{-8} N_{\mathrm{w}}$, and $N_{\mathrm{D}}=2.0$ $\times 10^{-14} N_{\mathrm{w}}$, as shown in Table I) and the best-fitted $N_{\mathrm{w}}$, the number density of hydration water $\left(=n_{\mathrm{h}} \sum N_{\mathrm{s}}\right)$ was calculated to be $0.0074 \pm 0.0005 / \AA^{3}$. In this framework, the fraction of hydration water to total intracellular water ( $\left.f_{\text {hyd }}=n_{\mathrm{h}} \sum N_{\mathrm{s}} / N_{\mathrm{w}}\right)$ was found to be $f_{\text {hyd }}=23.8 \pm 7.4 \%$ for intact HeLa cells. This result corresponds to an "average" hydration water amount of $1.25 \mathrm{~g} \mathrm{H}_{2} \mathrm{O} / \mathrm{g}$ solute in HeLa cell monolayer; roughly similar to that of lysozyme $\left(1.4 \mathrm{~g} \mathrm{H}_{2} \mathrm{O} / \mathrm{g}\right.$ protein) and corresponding to three hydration layers. ${ }^{44}$ Additionally, $8 \%$ smaller molecular radius of hydration

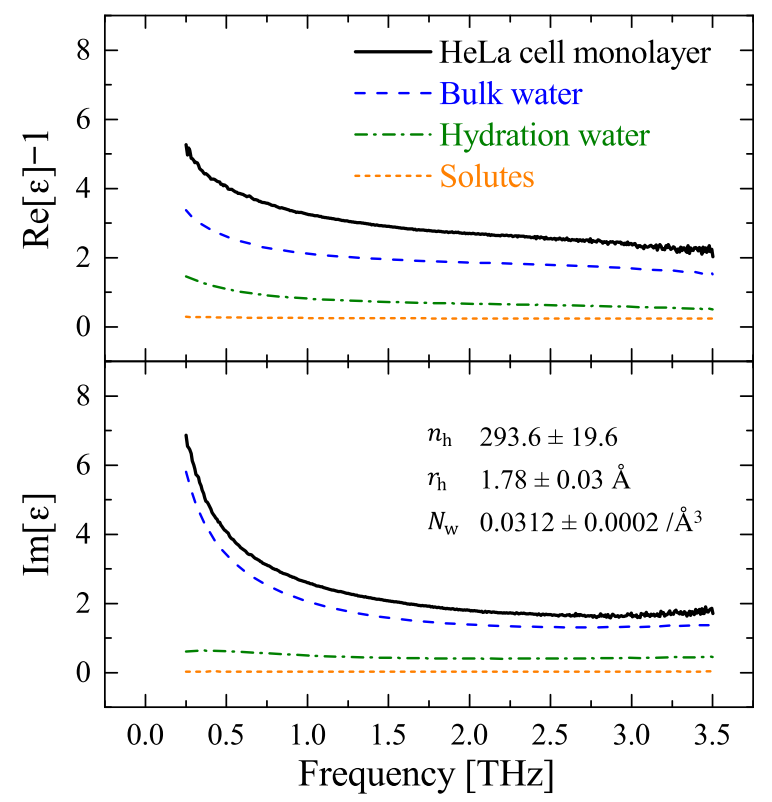

FIG. 3. Decomposition of $\tilde{\varepsilon}_{H e L a}(\omega)$ into the complex susceptibilities of bulk water $\tilde{\chi}_{\text {bulk }}(\omega)$, hydration water $\tilde{\chi}_{\text {hyd }}(\omega)$, and solutes $\tilde{\chi}_{\text {sol }}(\omega)$. The best-fitted parameters are shown in the bottom part. water, $r_{\mathrm{h}}=1.78 \AA$, than that of bulk water $\left(r_{\mathrm{w}}\right)$, was consistent with the fact that the dynamical motion of hydration water is slowed down. ${ }^{14}$ The relative effective volume of bulk water to hydration water, $\left(r_{\mathrm{w}} / r_{\mathrm{h}}\right)^{3} \approx 1.27$, suggests a higher density of intracellular hydration water than bulk water by a factor of $27 \%$.

Our estimation of $f_{\text {hyd }}$ for intact HeLa cells determined by $\mathrm{THz}$ spectroscopy was larger than that of monocellular organisms and human red blood cells (10\%-15\%) evaluated by $\mathrm{NMR}^{6}$ and $\mathrm{QENS},{ }^{8}$ but essentially equivalent to that of cardiac muscle tissue $(\sim 27 \%)$ estimated by INS. ${ }^{9}$ The relatively smaller $f_{\text {hyd }}$ of monocellular organisms ${ }^{6}$ and human red blood cells ${ }^{8}$ may stem from the different chemical components of the cell interior compared to human living cells; these cells are missing some of the organelles found in eukaryotic cells, such as the nuclear envelope and mitochondria, and thus the hydration mechanisms can be thought to be quite different from those in common human cells. Second, even though $f_{\text {hyd }}$ of "freshly excised" human cardiac muscle tissue ${ }^{9}$ was similar to that of our study, tissue dissection and enclosure processes before measurement make it difficult to access the "true" in vivo intracellular hydration state. Additionally, these measurements require preprocessing such as H/D substitution and cryogenic cooling, which imposes further modification of the intracellular environment. On the other hand, in our measurement, the hydration state of the intact HeLa cell monolayer was experimentally determined under physiological conditions with optimum temperature and $\mathrm{pH}$.

In this study, with the use of THz TD-ATR spectroscopy, combined with the application of the extended theory of Onsager, we determined the hydration state inside an intact HeLa cell monolayer. Our result indicates $23.8 \pm 7.4 \%$ of the HeLa intracellular water was bound to biomolecules (i.e., proteins, lipid, RNA, and DNA), which corresponds to $1.25 \mathrm{~g} \mathrm{H}_{2} \mathrm{O} / \mathrm{g}$ solute of hydration water. These intracellular hydration water molecules exhibited slowed down reorientation dynamics relative to that of bulk water. This in vivo characterization of intracellular hydration state under physiological conditions opens up the possibilities of further delving into the anomalous properties of cell water ${ }^{45}$ and offers various potential applications in clinical diagnosis and tissue engineering in the future.

We are grateful to Mr. Motoki Imamura and Mr. Akiyoshi Irisawa (ADVANTEST Corporation, Japan) for their technical supports. We also acknowledge Associate 
Professor Garry John Piller (Graduate School of Agriculture, Kyoto University, Japan) for his help and useful discussions. Financial support was provided by Industry-Academia Collaborative R\&D from Japan Science and Technology Agency (JST) and JSPS KAKENHI Grant No. 26295.

${ }^{1}$ M. Chaplin, Nat. Rev. Mol. Cell. Biol. 7, 861 (2006).

${ }^{2}$ P. B. Weisz, Science 179, 433 (1973).

${ }^{3}$ P. M. Wiggins, Cell Biol. Int. 20, 429 (1996).

${ }^{4}$ G. D. Fullerton, K. M. Kanal, and I. L. Cameron, Cell Biol. Int. 30, 86 (2006).

${ }^{5}$ R. Damadian, Science 171, 1151 (1971).

${ }^{6}$ E. Persson and B. Halle, Proc. Natl. Acad. Sci. U.S.A. 105, 6266 (2008).

${ }^{7}$ M. Tehei, B. Franzetti, K. Wood, F. Gabel, E. Fabiani, M. Jasnin, M. Zamponi, D. Oesterhelt, G. Zaccai, M. Ginzburg, and B.-Z. Ginzburg, Proc. Natl. Acad. Sci. U.S.A. 104, 766 (2007).

${ }^{8}$ A. M. Stadler, J. P. Embs, I. Digel, G. M. Artmann, T. Unruh, G. Büldt, and G. Zaccai, J. Am. Chem. Soc. 130, 16852 (2008).

${ }^{9}$ R. C. Ford, S. V. Ruffle, A. J. Ramirez-Cuesta, I. Michalarias, I. Beta, A. Miller, and J. Li, J. Am. Chem. Soc. 126, 4682 (2004).

${ }^{10}$ M. Jasnin, A. Stadler, M. Tehei, and G. Zaccai, Phys. Chem. Chem. Phys. 12, 10154 (2010).

${ }^{11}$ F. Sebastiani, A. Orecchini, A. Paciaroni, M. Jasnin, G. Zaccai, M. Moulin, M. Haertlein, A. De Francesco, C. Petrillo, and F. Sacchetti, Chem. Phys. 424, 84 (2013).

${ }^{12}$ E. O. Potma, W. P. de Boeij, and D. A. Wiersma, Biophys. J. 80, 3019 (2001).

${ }^{13}$ P. Ball, Chem. Phys. Chem. 9, 2677 (2008).

${ }^{14}$ T. Arikawa, M. Nagai, and K. Tanaka, Chem. Phys. Lett. 457, 12 (2008).

${ }^{15}$ M. Hishida and K. Tanakan, Phys. Rev. Lett. 106, 158102 (2011).

${ }^{16}$ K. Shiraga, T. Suzuki, N. Kondo, and Y. Ogawa, J. Chem. Phys. 141, 235103 (2014).

${ }^{17}$ K. Shiraga, H. Naito, T. Suzuki, N. Kondo, and Y. Ogawa, J. Phys. Chem. B 119, 5576 (2015)

${ }^{18}$ B. Bagchi, Chem. Rev. 105, 3197 (2005).

${ }^{19}$ K. Shiraga, Y. Ogawa, T. Suzuki, N. Kondo, A. Irisawa, and M. Imamura, Appl. Phys. Lett. 102, 053702 (2013).
${ }^{20}$ K. Shiraga, Y. Ogawa, T. Suzuki, N. Kondo, A. Irisawa, and M. Imamura, J. Infrared Millimeter, Terahertz Waves 35, 493 (2014).

${ }^{21}$ M. Nagai, H. Yada, T. Arikawa, and K. Tanaka, Int. J. Infrared Millimeter Waves 27, 505 (2006).

${ }^{22}$ P. U. Jepsen, D. G. Cooke, and M. Koch, Laser Photonics Rev. 5, 124 (2011).

${ }^{23}$ K. Ohta and R. Iwamoto, Appl. Spectrosc. 39, 418 (1985).

${ }^{24}$ S. Gabriel, R. W. Lau, and C. Gabriel, Phys. Med. Biol. 41, 2271 (1996).

${ }^{25} \mathrm{~B}$. Born and M. Havenith, J. Infrared Millimeter, Terahertz Waves 30, 1245 (2009)

${ }^{26}$ H. Yada, M. Nagai, and K. Tanaka, Chem. Phys. Lett. 473, 279 (2009).

${ }^{27}$ R. Gente, N. Born, N. Vo $\beta$, W. Sannemann, J. Léon, M. Koch, and E. Castro-Camus, J. Infrared Millimeter, Terahertz Waves 34, 316 (2013).

${ }^{28}$ T. Arikawa, M. Nagai, and K. Tanaka, Chem. Phys. Lett. 477, 95 (2009).

${ }^{29}$ D. N. Wheatley, M. S Inglis, M. A. Foster, and J. E. Rimington, J. Cell Sci. 88, 13 (1987).

${ }^{30}$ R. T. Dell'orco and G. Melnykovych, J. Cell. Physiol. 76, 101 (1970).

${ }^{31}$ K. Park, J. Jang, D. Irimia, J. Sturgis, J. Lee, J. P. Robinson, M. Toner, and R. Bashir, Lab Chip 8, 1034 (2008).

${ }^{32}$ J. O. Bishop, J. G. Morton, M. Rosbash, and M. Richardson, Nature 250, 199 (1974).

${ }^{33}$ G. G. Maul and L. Deaven, J. Cell Biol. 73, 748 (1977).

${ }^{34}$ K. B. Hendil, R. Hartmann-Petersen, and K. Tanaka, J. Mol. Biol. 315, 627 (2002).

${ }^{35}$ B. Alberts, D. Bray, K. Hopkin, A. Johnson, J. Lewis, M. Raff, K. Roberts, and P. Walter, Essential Cell Biology, 2nd ed. (Garland Science, 2005).

${ }^{36}$ B. Lewin, Genes VIII (Pearson education, Inc., 2004), p. 135.

${ }^{37}$ A. Dobi, M. A. Mahan, and D. V. Agoston, Electrophoresis 18, 12 (1997).

${ }^{38}$ H. P. Erickson, Biol. Proced. Online 11, 32 (2009).

${ }^{39}$ S. M. Johnson and N. Buttress, Biochim. Biophys. Acta 307, 20 (1973).

${ }^{40} \mathrm{P}$. Tiollais, F. Galibert, and M. Boiron, Eur. J. Biochem. 18, 35 (1971).

${ }^{41}$ P. Volpe and T. Eremenko, Eur. J. Biochem. 32, 227 (1973).

${ }^{42}$ A. G. Markelz, A. Roitberg, and E. J. Heilweil, Chem. Phys. Lett. 320, 42 (2000).

${ }^{43}$ C. Zhang, E. Tarhan, A. K. Ramdas, A. M. Weiner, and S. M. Durbin, J. Phys. Chem. B 108, 10077 (2004).

${ }^{44}$ G. D. Fullerton, V. A. Ord, and I. L. Cameron, BBA-Protein Struct. M. 869, 230 (1986).

${ }^{45}$ K. Luby-Phelps, Mol. Biol. Cell 24, 2593 (2013). 